GEOPHYSICAL RESEARCH LETTERS, VOL. 16, NO. 3, PAGES 259-262, MARCH 1989

\title{
MEASURING COMPLEX SPECTRA OF LONG-PERIOD SURFACE WAVES FOR EARTHQUAKE SOURCE ANALYSIS
}

\author{
Jiajun Zhang and Thome Lay
}

Department of Geological Sciences, The University of Michigan

\begin{abstract}
Long-period surface waves from large earthquakes can provide reliable estimates of the source depth, rupture duration, seismic moment, and fault orientation. This requires accurate surface wave phase and amplitude spectra and precise propagation corrections. Standard procedures for estimating spectra of traveling waves in source analyses are inaccurate for periods exceeding $300 \mathrm{~s}$, so the longer periods are usually analyzed by free oscillation analysis if at all. We apply a multiple filter analysis to extract complex spectra for periods up to $500 \mathrm{~s}$ suitable for traveling wave spectral inversions. This will allow improved constraints on the rupture process of large earthquakes to be obtained by inversion methods using a single formalism.
\end{abstract}

\section{Introduction}

Long-period Rayleigh and Love waves have been extensively used in analysis of mantle structure and source parameters of large earthquakes. Following the approach introduced by Satô [1958], various techniques have recently been developed for mapping aspherical heterogeneity of the upper mantle [e.g., Nakanishi and Anderson, 1983] and for inverting observations for source characteristics [e.g., Kanamori and Given, 1981; Zhang and Kanamori, 1988]. These procedures require spectra of surface waves, which are obtained by Fourier analysis. Typically, the spectra are determined for a specified group velocity window short enough to isolate the signal from interference by successive great circle passages, but long enough to reduce leakage while yielding the desired frequency resolution. However, there is no guarantee that an appropriate window can be found when waves of different modes of propagation are present. The multitaper method of Thomson [1982] gives estimates with less leakage than direct estimates; however, the former method requires lengthy computations when a large data set is to be analyzed. For very long-period (greater than $300 \mathrm{~s}$ ) waves, errors in direct estimates are often very large, because the waves are strongly dispersive and isolation of the signal is difficult. As a result, global aspherical earth structure and seismic source investigations using surface wave spectra have been restricted to periods less than $300 \mathrm{~s}$.

Several dispersed waveform analysis techniques are routinely used for extracting phase and group velocities for surface waves. Curiously, while these techniques have been applied to analyze earth structure from sequential passes of global circuiting waves [e.g., Dziewonski et al., 1972] or to analyze surface waves with periods less than $100 \mathrm{~s}$ [Russell et al., 1988], they are not routinely used in large earthquake source investigations. Because of the importance of the long-period surface waves for constraining large earthquake source parameters, particularly rupture duration and seismic moment, we have adapted the dispersion analysis techniques to the problem of retrieving complex spectra for long-period surface waves using a single formalism.

Copyright 1989 by the American Geophysical Union.

Paper number 89GL00217.

$0094-8276 / 89 / 89 \mathrm{GL}-00217 \$ 03.00$

\section{Method}

The procedure that we use is essentially the residual dispersion method of Dziewonski et al. [1972] modified to include the source terms. This procedure transforms a dispersed wave train to a pulse-like signal by cross-correlating the observation with a reference signal having similar dispersion. The reference signal, which we will call an isolation filter, can be made to have exactly the same dispersion as the observation either by performing an autocorrelation or by iteration [Dziewonski and Hales, 1972; Herrin and Goforth, 1977]. Various strategies for filtering the resulting "pseudo autocorrelation function" for the purpose of reducing multipathing contamination have been examined [Russell et al., 1988]. The cross-correlation function can then be processed by multiple filtering [Dziewonski et al., 1969] to isolate individual fundamental or overtone modes for given frequencies and their corresponding group and phase velocities.

Following Kanamori and Given [1981], the spectrum of surface-wave displacements caused by an earthquake for a station at azimuth $\phi$ and epicentral distance $r$ can be calculated by

$$
\begin{array}{r}
G(\omega)=S(\omega, \phi) \exp (-i k r) \exp (i m \pi / 2) \exp \left(\phi_{l}\right) \\
\exp (-\omega r / 2 Q U) /(\sin (r / R))^{1 / 2}
\end{array}
$$

where $k(\omega)$ is the wave number, $\mathrm{m}$ the number of the polar and antipodal passages, $\phi_{i}$ the initial phase at the source, $R$ the radius of the earth, $U$ the group velocity, $Q$ the quality factor, and $S(\omega, \phi)$ the complex source spectrum. Let

$$
\begin{aligned}
& x_{n}(t)=\frac{1}{2 \pi} \int_{-\infty}^{\infty} G(\omega)\left\{\exp \left[-\alpha\left[\frac{\omega-\omega_{n}}{\omega_{n}}\right]^{2}\right]\right. \\
& \left.+\exp \left[-\alpha\left[\frac{\omega+\omega_{n}}{\omega_{n}}\right]^{2}\right]\right\} W(\omega) \exp (i \omega t) d \omega
\end{aligned}
$$

represent a signal obtained from the displacements by application of a multiple filter. Here $W(\omega)$ is an isolation filter, $\omega_{n}$ the center frequency of the $n$th Gaussian filter with bandwidth controlled by the parameter $\alpha$. If $W(\omega)$ is the Fourier transform of a real time function, then $x_{n}(t)$ is real. If we write

$$
M(\omega)=G(\omega) W(\omega)=A_{m}(\omega) \exp \left(i \phi_{m}(\omega)\right)
$$

and assume

$$
\begin{aligned}
& A_{m}(\omega)=A_{m}\left(\omega_{n}\right)+A_{m}^{\prime}\left(\omega_{n}\right)\left(\omega-\omega_{n}\right) \\
& \phi_{m}(\omega)=\phi_{m}\left(\omega_{n}\right)+\phi_{m}^{\prime}\left(\omega_{n}\right)\left(\omega-\omega_{n}\right)
\end{aligned}
$$

the integral (2), and its instantaneous amplitude (envelope) and phase can be evaluated as in Dziewonski et al. [1972]. The height of the envelope is given by

$$
A_{n}=\frac{\omega_{n}}{\sqrt{\pi \alpha}} A_{m}\left(\omega_{n}\right)
$$

which corresponds to the lag time $t_{n}=-\phi_{m}^{\prime}\left(\omega_{n}\right)$ and phase

$$
\Phi_{n}=\phi_{m}\left(\omega_{n}\right)+\omega_{n} t_{n}
$$

Therefore, if $M(\omega)$ satisfies (4) and (5), the amplitude spectra, $A_{g}(\omega)$, and phase spectra, $\phi_{g}(\omega)$, of the displacements can be evaluated by 


$$
\begin{gathered}
A_{g}(\omega)=\frac{\sqrt{\pi \alpha}}{\omega_{n}} A_{n} / A_{W}(\omega) \\
\phi_{g}(\omega)=\Phi_{n}-\omega_{n} t_{n}-\phi_{W}\left(\omega_{n}\right)
\end{gathered}
$$

where $A_{W}(\omega)$ and $\phi_{W}(\omega)$ are the amplitude and phase spectra of $W(\omega)$, respectively. If we write

$$
S(\omega, \phi)=A_{s}(\omega, \phi) \exp \left(i \phi_{s}(\omega, \phi)\right)
$$

and assume

$$
\begin{gathered}
A_{W}=\hat{A}_{s}^{-1}(\omega, \phi) \exp (\omega r / 2 \hat{Q} \hat{U}) \\
\phi_{W}=-\hat{\phi}_{s}(\omega, \phi)+\hat{k} r
\end{gathered}
$$

where $\hat{A_{s}}=A_{s}, \hat{Q}=Q, \hat{U}=U, \hat{\phi}_{s}=\phi_{s}, \hat{k}=k$, then $M(\omega)$ satisfies (4) and (5).

\section{Numerical Test}

While the original residual dispersion method of Dziewonski et al. [1972] clearly provides improved group velocity estimates compared to methods lacking an isolation filter, it was not known how much improvement in phase and amplitude measurements could be achieved using our similar procedure relative to the standard direct method of spectral estimation from a truncated signal. We will show that our procedure has significant advantages over the direct method for Rayleigh waves with periods longer than $300 \mathrm{~s}$.

Our primary concem is to assess the error of estimated amplitude and phase spectra, which depends on the nature of the dispersion and interference of various wave trains. We tested the multiple filter method (MF) described above using many synthetic seismograms, for which the spectra are known. We first establish the potential accuracy of the method using only noise free fundamental mode waves, and then summarize the results of many other calculations with realistic overtone noise contamination to provide guidance for the practical application of our technique.

A record for station GAR of the International Deployment of Accelerometers (IDA) network was computed for a hypothetical earthquake with a step-function point source in the Tonga region for earth model PREM [Dziewonski and Anderson, 1981]. Fundamental modes with periods greater than $45 \mathrm{~s}$ were included, and the method of Kanamori and Cipar [1974] was used for the computation. The time sampling increment was $20 \mathrm{~s}$. The station has azimuth, $\phi=302^{\circ} .42$, and distance, $\theta=122^{\circ} .19$, from the source. Figure 1a shows a filtered segment of the record (including the instrument response) with Rayleigh waves propagating along the minor arc $\left(R_{1}\right)$ and major arc $\left(R_{2}\right)$ of the great circle. We measured the amplitude and phase spectra of periods from 80 to $800 \mathrm{~s}$ of the $R_{1}$ wave train. The Rayleigh waves in this period range correspond to spheroidal modes of angular orders from 123 to 7 , spanning most of the energy of Rayleigh waves radiated from an earthquake source.

We used the isolation filter: $A_{W}=1$ and $\phi_{W}=\hat{k} r$, which does not require a priori information about the source, and is the same as that used by Dziewonski et al. [1972]. This filter is a special case of the more general technique, which simultaneously matches both amplitudes and phase caused by source radiation and propagation as in equations (11) and (12). Full utilization of (11) and (12) can improve the accuracy of the measurements if the source is known. However, even with the simple phase match filter, we found that the advantages of the MF over direct methods can be demonstrated.

Here $\hat{k}$ is chosen for the average ocean model of Regan and Anderson [1984] (hereinafter referred to as R-A). For the period range concerned, the amplitude $A_{s}(\omega)$ and phase $\phi_{s}(\omega)$ of the source and the effect of attenuation $\exp (\omega r / 2 \hat{Q} \hat{U})$ can be considered to be slowly varying functions of frequency, and the wave number for R-A is about the same as that for PREM.

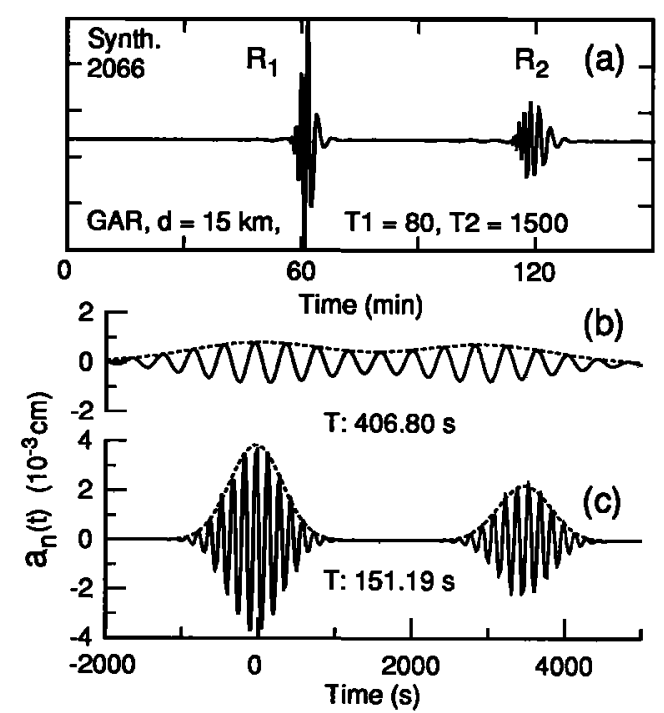

Fig. 1. (a) Synthetic vertical component record (counts) for GAR $\left(39^{\circ} \mathrm{N}, 70^{\circ} 30 \mathrm{E}\right)$ for an earthquake in the Tonga region $\left(22^{\circ} 88 \mathrm{~S}, 175^{\circ} 9 \mathrm{~W}\right.$, depth $\left.15 \mathrm{~km}\right)$ with seismic moment, $10^{27}$ dyne $\mathrm{cm}$, strike, $\phi=180^{\circ}$, dip, $\delta=45^{\circ}$, and slip, $\lambda=90^{\circ}$. The trace is filtered using a Gaussian filter with a passband from 80 to $1500 \mathrm{~s}$ and plotted from the origin time. The signals of ground displacements (solid line) and their envelopes (dashed line) for the periods of $406.8 \mathrm{~s}$ (b) and $151.19 \mathrm{~s}$ (c) obtained from the synthetic record by applying the MF with $\alpha=100$ and relative bandwidth of 0.30 for the Gaussian filter.

Therefore, (4) and (5) are satisfied to the first order, which is critical for the success of the residual dispersion method.

The synthetic recond of the first $150 \mathrm{~min}$ after the origin time was padded with the end value to a total of 1024 data points, and windowed by an $20 \%$ cosine taper to reduce leakage at high frequencies. The tapered record was then Fourier transformed, corrected for the instrument response, multiplied by the isolation filter, and further filtered for each central frequency by a Gaussian band-pass filter with a given $\alpha$. The Gaussian filter is truncated symmetrically to avoid unnecessary computations; the ratio between the half bandwidth and the central frequency gives the relative bandwidth of the filter. In this test, we use Gaussian filters truncated at lengths at which the response dropped at least by $30 \mathrm{db}$ from the maximum.

We calculated the inverse Fourier transforms of the filtered spectra and $\pi / 2$ phase shifted spectra to obtain the time signal $x_{n}(t)$ and its Hilbert transform; and then calculated the instantaneous amplitude and phase for the central frequency. Figures $1 \mathrm{~b}$ and $1 c$ show $x_{n}(t)$ and their envelopes for two periods. Each envelope has two maxima, corresponding to $R_{1}$ and $R_{2}$, respectively. The isolated mode cross-correlograms show almost zero lag times for the phase equalized $R_{1}$ wave trains. For every frequency, $\omega_{n}$, we obtained the maximum amplitude $A_{n}$ of the envelope, corresponding instantaneous phase $\Phi_{n}$, and lag time $t_{n}$. We then calculated the amplitude and phase spectrum of the displacements for each frequency using (8) and (9).

The parameter $\alpha$ controls the resolution of the maximum of the envelope. Filters with small $\alpha$ have lower resolution in frequency and higher resolution in time than filters with large $\alpha$. For strongly dispersed waves, such as Rayleigh waves of periods greater than $400 \mathrm{~s}$, time domain resolution is essential, thus small $\alpha$ is more appropriate. However, for weakly dispersed waves, such as Rayleigh waves of periods around $220 \mathrm{~s}$, large $\alpha$ is more suitable.

Figure 2 compares the spectral estimates of the displacements obtained from the synthetic record by deconvolving the instru- 


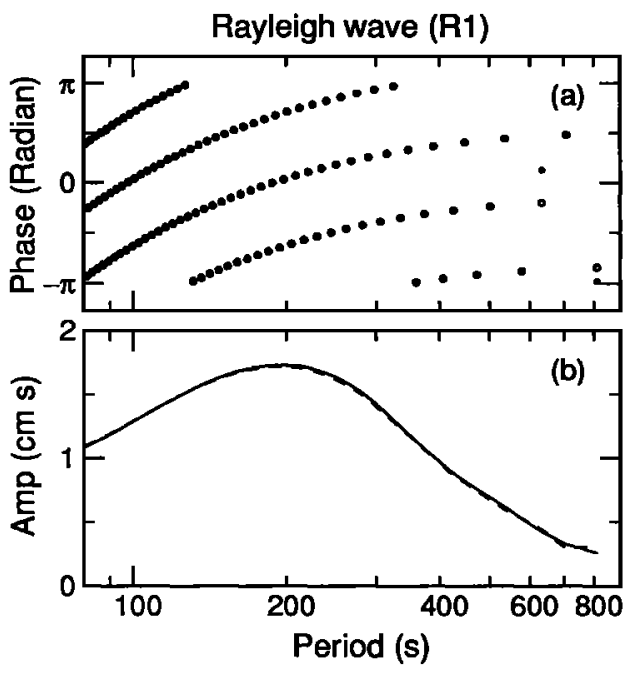

Fig. 2. Phase (a) and amplitude (b) spectra of displacements for $\mathrm{R}_{1}$ : predicted phase (open circles), measured phase (solid circles), predicted amplitude (solid line), and measured amplitude (dashed line). Measured spectra are obtained using the MF; the Gaussian filters have relative bandwidth of .3 and $\alpha=160$ for periods from 80 to $400 \mathrm{~s}$, and $\alpha=40$ for periods from 400 to $800 \mathrm{~s}$.

ment response and applying the MF with the predicted spectra computed using the method of Kanamori and Given [1981]. Since phase spectra vary rapidly with period, they are plotted for each mode. Different $\alpha$ values were chosen for different periods to obtain satisfactory resolution. For periods up to $600 \mathrm{~s}$, measured values of both amplitude and phase agree with predicted values reasonably well. However, for periods longer than $600 \mathrm{~s}$, where the signal power is relatively small, relative errors for both amplitude and phase are large.

In the direct method of spectral estimation a tapered segment of the original signal, which is treated as periodic, is Fourier transformed. The position, width and shape of the taper are chosen empirically to obtain sufficient spectral resolution according to the nature of the dispersed signal, which depends primarily on the propagation distance of the waves and the quality of the record. The position and width of the taper are determined by a fixed or moving group velocity window.

To compare the spectra obtained by the MF with the results by the direct method, we measured the spectra of $R_{1}$ using either fixed or moving group velocity window, and a $20 \%$ cosine taper. The fixed window: $2.93-4.46 \mathrm{~km} \mathrm{~s}^{-1}$, which is commonly used in analyses of $R_{1}$ mantle Rayleigh waves, was applied only to periods shorter than $300 \mathrm{~s}$; much of the wave energy for longer periods is not contained in this window. The moving window is chosen using the group velocity curve of PREM. For each period, the window is centered at the group arrival time, and is 10 times longer than the period. The window is adjusted when necessary to avoid interference between $R_{1}$ and $R_{2}$.

Figure 3a shows relative amplitude errors, which are differences between measured and predicted values normalized by the predicted values, for the MF and direct method for periods from 80 to $650 \mathrm{~s}$. For periods shorter than $300 \mathrm{~s}$, all methods used here give errors less than $5 \%$. For periods longer than 350 s, errors for the direct method and for the MF with $\alpha=100$ become very large, while errors for the MF with $\alpha=\mathbf{4 0}$ remain less than $5 \%$. Figure $3 b$ shows phase errors. For periods shorter than $300 \mathrm{~s}$, all methods give errors less than 0.1 radian. For periods from 350 to $600 \mathrm{~s}$, the errors for the direct method and for the MF with $\alpha=100$ become very large, while errors for the MF with $\alpha=40$ remain less than 0.1 radian.

Figure $4 \mathrm{a}$ shows relative amplitude errors for the MF with Gaussian filters of various values of $\alpha$ for periods from 80 to 400

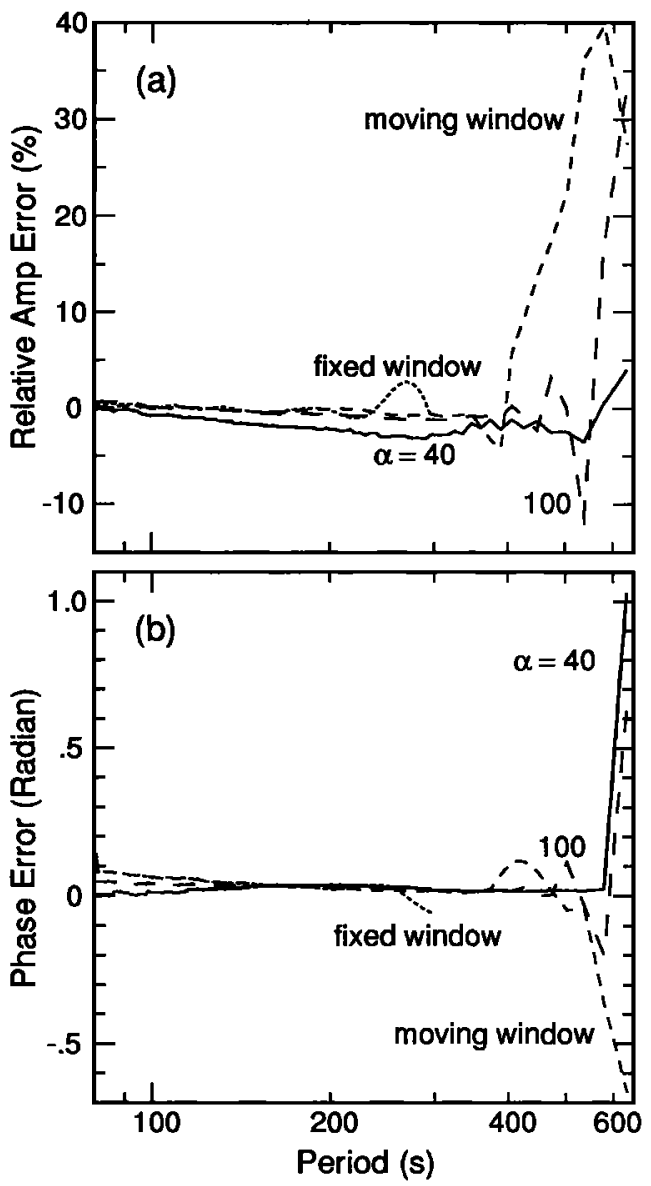

Fig. 3. Relative errors (in percentage) of amplitude spectra (a) and errors (in radian) of phase spectra (b) obtained by the MF with $\alpha=40$ (solid line) and $\alpha=100$ (long dashed line) for Gaussian filters, and by a fixed group velocity window (dotted line), and a moving window (short dashed line). The fixed velocity window is applied only to periods from 80 to $300 \mathrm{~s}$.

s. The case with $\alpha=300$, which has the highest frequency resolution among all MF used here, gives good results for periods up to about $250 \mathrm{~s}$. There appears to be a long-period limit for each MF used, beyond which the errors increase rapidly. This limit increases as $\alpha$ decreases. For periods from 80 to $400 \mathrm{~s}$, the MF with $\alpha=100$ gives the best overall estimates. Figure $4 \mathrm{~b}$ shows phase errors obtained by the MF with various Gaussian filters. The MF with $\alpha$ from 40 to 100 give overall better estimates than other MF considered.

The noise free test described above demonstrates that while direct methods break down for periods above $300 \mathrm{~s}$ the MF method can give sufficiently accurate results out to $500 \mathrm{~s}$, even when the simple isolation filter is used. Given the small scatter in dispersion observed at very long period, standard reference earth models provide adequate isolation filters.

Many additional tests were made to explore the practical utilization of the MF procedure in the study of large earthquakes. Synthetic data sets for various distances and noise characteristics were analyzed. We computed seismograms including all fundamental and overtone modes with periods greater than $45 \mathrm{~s}$ for the earth model 1066a [Gilbert and Dziewonski, 1975] for various distances. The MF procedure was then used to measure amplitude and phase of fundamental mode Rayleigh waves. The overtones provide realistic noise levels, similar to most observational data sets. 


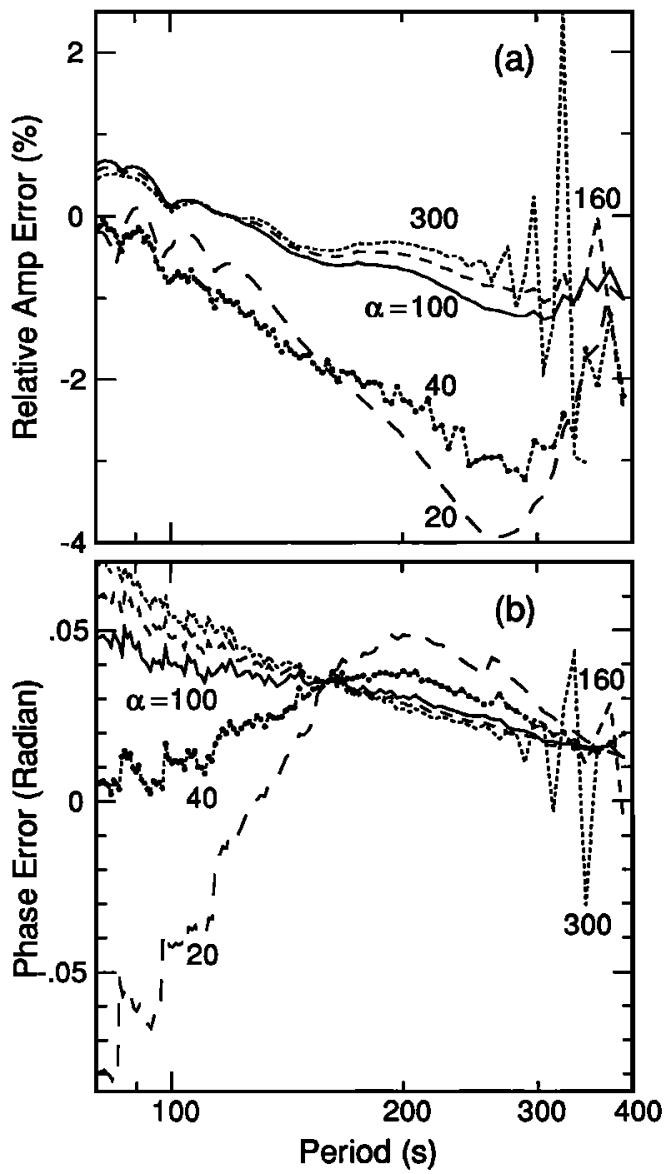

Fig. 4. Relative errors (in percentage) of amplitude spectra (a) and errors (in radian) of phase spectra (b) obtained by the MF with Gaussian filters of various values of $\alpha$.

The results confirm the conclusion by Nakanishi and Anderson [1983] that higher-mode interference does not usually cause systematic biases for the measurements of fundamental mode Rayleigh waves. However, interference between fundamental mode waves propagating in opposite directions is the main source of error. The MF method works best for intermediate distances $\left(40^{\circ}\right.$ to $\left.140^{\circ}\right)$, at which the waves are well dispersed and $R_{1}$ and $R_{2}$ are well separated. For $R_{1}$ arrivals in this range, the MF gives measurements of sufficient accuracy (amplitude errors less than $5 \%$, phase errors less than 0.1 radian) for the period range 80 to $500 \mathrm{~s}$; while the direct method gives results with similar accuracy only for periods up to $250-300 \mathrm{~s}$.

At distances closer to the source or its antipode, the errors become larger. For a distance of $30^{\circ}$, at periods from 80 to 500 $s$, the MF gives errors less than $10 \%$ but usually larger than $5 \%$ for amplitudes and less that 0.1 radian for phase; for periods from 80 to $260 \mathrm{~s}$, the direct method gives errors less than $10 \%$ for amplitudes and less than $\mathbf{0 . 2}$ radian for phase. For a distance of $150^{\circ}$, at periods from 80 to $500 \mathrm{~s}$, the MF gives errors less than $10 \%$ but usually larger than $5 \%$ for amplitudes and less than 0.3 radian but usually larger than 0.1 radian for phase; the direct method has similar accuracy only for periods from 80 to $210 \mathrm{~s}$.

\section{Discussion and Conclusions}

We apply a multiple filter method to measure the complex spectrum of long-period surface waves. Numerical tests using synthetic seismograms comprised of fundamental mode and over- tone Rayleigh waves indicates that this method gives accurate spectral estimates for periods as long as $500 \mathrm{~s}$. This will enable traveling wave inversions for source and aspherical structure to be extended beyond the current limit of $300 \mathrm{~s}$ imposed by current spectral estimation practices.

The accuracy of the method depends on how closely the isolation filter approximates the dispersion of the observed surface waves. As a first approximation the dispersion curve of a global earth model can be used to construct an adequate isolation filter for the multiple filter method for periods greater than $100 \mathrm{~s}$. This allows rapid analysis of many observations, which is essential for source and structure inversions. Further analysis of the various parameters in the multiple filter method, such as the isolation and band-pass filters, is expected to improve the method for the long-period surface wave application.

Acknowledgments. We thank the reviewers for helpful comments. This work was supported by NSF grant EAR-8451715.

\section{References}

Dziewonski, A. M., and D. L. Anderson, Preliminary reference Earth model, Phys. Earth and Planet. Int., 25, 297-356, 1981.

Dziewonski, A. M., and A. L. Hales, Numerical analysis of dispersed seismic waves, in Methods of Computational Physics, 11, edited by B. A. Bolt, pp. 39-85, Academic Press, New York, 1972.

Dziewonski, A., S. Bloch, and M. Landisman, A technique for the analysis of transient seismic signals, Bull. Seismol. Soc. Am., 59, 427-444, 1969.

Dziewonski, A., J. Mills, and S. Bloch, Residual dispersion measurement-A new method of surface wave analysis, Bull. Seismol. Soc. Am., 62, 129-139,1972.

Gilbert, F. and A. M. Dziewonski, An application of normal mode theory to the retrieval of structure parameters and source mechanisms from seismic spectra, Phil. Trans. R. Soc. Lond. A., 278, 187-269, 1975.

Herrin, E., and T. Goforth, Phase-matched filters: application to the study of Rayleigh waves, Bull. Seismol. Soc. Am., 67, 1259-1275, 1977.

Kanamori, H., and J. J. Cipar, Focal process of the great Chilean earthquake, May 22, 1960, Phys. Earth Planet. Inter., 9, 128$136,1974$.

Kanamori, H., and J. W. Given, Use of long-period surface waves for rapid determination of earthquake-source parameters, Phys. Earth Planet. Inter., 27, 8-31, 1981.

Nakanishi, I., and D. L. Anderson, Measurements of mantle wave velocities and inversion for lateral heterogeneity and anisotropy, I, Analysis of great circle phase velocities, J. Geophys. Res., 88, 10,267-10,283, 1983.

Regan, J., and D. L. Anderson, Anisotropic models of the upper mantle, Phys. Earth Planet. Inter., 35, 227-263, 1984.

Russell, D, R., R. B. Herrmann, and H.-J. Hwang, Application of frequency variable filters to surface-wave amplitude analysis, Bull. Seismol. Soc. Am., 78, 339-354, 1988.

Satô, Y., Attenuation, dispersion, and the wave guide of the $\mathbf{G}$ wave, Bull. Seismol. Soc. Am., 48, 231-251,1958.

Thomson, D. J., Spectrum estimation and harmonic analysis, IEEE Proc., 70, 1055-1096, 1982.

Zhang, J., and H. Kanamori, Depths of large earthquakes determined from long-period Rayleigh waves, J. Geophys. Res., 93, 4850-4868, 1988.

J. Zhang and T. Lay, Department of Geological Sciences, The University of Michigan, Ann Arbor, MI 48109.

(Received: November 7, 1988;

Revised: January 23, 1989;

Accepted: January 27, 1989) 